\title{
Relacionamento terapêutico e ensino de enfermagem psiquiátrica e saúde mental: tendências no Estado de São Paulo
}

\author{
THERAPEUTIC RELATIONSHIP AND TEACHING OF MENTAL HEALTH PSYCHIATRIC NURSING: \\ TENDENCIES IN THE STATE OF SÃO PAULO
}

RELACIÓN TERAPÉUTICA ENSEÑANZADE ENFERMERÍA PSIQUIÁTRICAY SALUD MENTAL: ACTUALIDADES EN EL ESTADO DE SÃO PAULO

\author{
Luciane Prado Kantorski', Leandro Barbosa de Pinho², \\ Toyoko Saeki ${ }^{3}$, Maria Conceição Bernardo de Mello e Souza ${ }^{4}$
}

\begin{abstract}
RESUMO
Este estudo apresenta um panorama sobre a inserção da temática do relacionamento terapêutico e da comunicação terapêutica no ensino de graduação em enfermagem, mais particularmente nas universidades públicas do Estado de São Paulo. Trata-se de uma pesquisa qualitativa e descritivoanalítica. O instrumento de coleta de dados foi um questionário aplicado a professores da área de enfermagem psiquiátrica e saúde mental das nove escolas do Estado, sendo que oito devolveram o questionário preenchido. Os dados foram agrupados levando-se em consideração a instituição, as disciplinas, os conteúdos abordados, os objetivos das atividades práticas e campos práticos. Verificamos que a maioria das escolas aborda o relacionamento terapêutico em suas propostas de ensino, configurando-se como uma possibilidade de se resgatar a enfermagem enquanto profissão que se preocupa com o ser humano em sua complexidade, em que o saberfazer preconizado é direcionado ao respeito das limitações, potencialidades, necessidades e relações interpessoais dos pacientes.
\end{abstract}

\section{DESCRITORES}

Enfermagem psiquiátrica.

Ensino.

Saúde mental.

\begin{abstract}
This study presents a panorama of the insertion of the thematic of therapeutic relationship and therapeutic communication in undergraduate courses in Nursing in the public universities of the State of São Paulo. This is a qualitative and descriptiveanalytical research. Data was collected through a questionnaire applied on professors of the area of Psychiatric Nursing and Mental Health from 8 schools and grouped according to the institution, the disciplines, the program and the objectives of the practical activities and practical fields. It was verified that most schools include the therapeutic relationship in their teaching approach, which makes possible the rescue of Nursing as a profession that is concerned with the human being in his/her complexity, in which the know-how is directed to the respect of the patients' limitations, potentialities, needs and interpersonal relations.
\end{abstract}

\section{KEY WORDS}

Psychiatric nursing.

Teaching.

Mental health.

\section{RESUMEN}

Este estudio presenta un panorama sobre la inserción de la temática de la relación y comunicación terapéutica, en la enseñanza del pre grado en enfermería particularmente en las universidades públicas del Estado de São Paulo. Se trata de una investigación cualitativa de tipo descriptivo-analítica. El instrumento de recolección de datos fue un cuestionario aplicado a profesores del área de enfermería psiquiátrica y salud mental de las nueve escuelas del Estado, de las cuales ocho devolvieron el cuestionario llenado. Los datos fueron agrupados llevándose en consideración la institución, las disciplinas, los contenidos abordados, los objetivos de las actividades prácticas y campos prácticos. Verificamos que la mayoría de las escuelas aborda la relación terapéutica en sus propuestas de enseñanza, configurándose como una posibilidad de rescatarse la enfermería en cuanto profesión que se preocupa por el ser humano en su complejidad, en donde el saber hacer preconizado es orientado hacia el respeto de las limitaciones, potencialidades, necesidades y relaciones interpersonales de los pacientes.

\section{DESCRIPTORES}

Enfermería psiquiátrica.

Enseñanza.

Salud mental.
1 Docente da Faculdade de Enfermagem e Obstetrícia da Universidade Federal de Pelotas / RS. Doutora em Enfermagem. kantorski@uol.com.br. Apoio CNPq.

2 Enfermeiro. Mestrando do Programa de PósGraduação em Enfermagem da Universidade Federal de Santa Catarina - UFSC. Apoio CNPq. lbpinho@uol.com.br 3 Doutora em Enfermagem. Docente do Departamento de Enfermagem Psiquiátrica e Ciências Humanas da Escola de Enfermagem de Ribeirão Preto da Universidade de São Paulo (EERP-USP).

4 Doutora em Enfermagem. Docente do Departamento de Enfermagem Psiquiátrica e Ciências Humanas da EERP-USP. consouza@eerp.usp.br 
Luciane Prado Kantorski

Leandro Barbosa de Pinho

Toyoko Saeki

Maria Conceição B.M. Souza

\section{INTRODUÇÃO}

A enfermagem lida com a dor e o sofrimento do ser humano desde que se conhece como enfermagem. É inerente à profissão o cuidado prestado ao indivíduo e à sua família de maneira a garantir-lhes boas condições de saúde física e mental e permitir o autodescobrimento de mecanismos de enfrentamento das adversidades, da dor e do sofrimento que determinadas ocasiões impõem.

Sofrer vai além do saber e do plano físico. Ele se caracteriza como uma junção de vertentes éticas, morais, religiosas, psicológicas, sociais e culturais. O sofrimento é mais que uma simples ameaça à integridade biológica, mas também à sua integridade como homem, como sujeito de ação, de reação e que possui necessidades próprias, específicas, que precisa de atenção e cuidado solidário ${ }^{(1)}$.

Nessa perspectiva, inserimos o sofrimento psíquico, enquanto construto da área da saúde mental. Sua complexidade nos permite ir além de nossa interpretação cognitiva. É um processo subjetivo, social, além de cultural, e suas dimensões atingem não só àquele que sofre como também a quem o rodeia. É uma condição em que a pessoa pode não interagir com a realidade objetiva das demais, tornar-se enfraquecida e, até mesmo, questionar o sentido da própria vida.

Uma das características da área da saúde mental foi exatamente a de buscar uma interpretação precisa à complexidade do adoecimento psíquico. A constituição da Psiquiatria pineliana do século XIX consiste no marco inicial, que aglutinou todas as formas de sofrimento psíquico e as caracterizou como doença mental. A tecnologia de ponta nessa área, atualmente, tem sido a relação entre sujeitos, a necessidade de se focar as atenções ao sofrimento da pessoa, que tem histórias singulares, irreproduzíveis, intransferíveis, bem como as possibilidades terapêuticas aplicáveis em cada caso ${ }^{(2)}$.

A psicanálise e a reforma psiquiátrica foram movimentos importantes contra o reducionismo da psiquiatria clássica, que caracterizava qualquer manifestação psíquica como doença mental. A psiquiatria preventiva ${ }^{(3)}$ foi um desses movimentos que se encarregou de centrar suas atenções no cliente e não em sua doença. As práticas terapêuticas da psiquiatria foram redimensio-nadas ao considerar o sofrimento psíquico como multifatorial, não tendo enfoque exclusivamente biomédico, mas também social, psicológico, político e cultural.
A enfermagem procura, desde seu surgimento enquanto profissão, acompanhar essas mudanças e redimensionar suas finalidades terapêuticas, com o auxílio das mais diversas áreas do conhecimento, como filosofia, sociologia, psicologia e antropologia. Elas têm fornecido subsídios teórico-práticos à complexidade que essas mudanças trouxeram. Permitem a (re) construção do conceito de ser humano, como também disponibilizam as diferentes possibilidades de se trabalhar com ele, visando à promoção de sua saúde integral ${ }^{(4)}$.

Consideramos o relacionamento terapêutico como um desses instrumentos de cuidado que permitem essa reintegração e reorganização da pessoa que padece psiquicamente. Trata-se de uma tecnologia de cuidado que possui um rol de saberes e práticas destinadas ao entendimento do ser humano em sua totalidade, de suas limitações, possibilidades, necessidades imediatas e potencialidades. Permite a reflexão, o crescimento pessoal, o reconhecimento do ser humano como importante promotor do cuidado de si e o desenvolvimento de habilidades para o enfrentamento do sofrimento e da reintegração social.

As tendências atuais das práticas terapêuticas centradas na pessoa, ressaltando o relacionamento terapêutico e a comunicação terapêutica, foram, aos poucos, incorporadas ao ensino de enfermagem psiquiátrica das escolas de enfermagem brasileiras, sendo ressaltadas em diferentes estudos ${ }^{(5-7)}$.

Em estudo sobre a inserção do relacionamento terapêutico na produção científica da enfermagem psiquiátrica e saúde mental, constatouse que existe uma preocupação dos enfermeiros em relatar suas experiências e desafios no cuidado a pacientes tendo as relações humanas como subsídio. Mencionou-se que a universidade, enquanto órgão formador de novos enfermeiros, tem papel fundamental no preparo teóricoprático desse profissional para a utilização do relacionamento terapêutico e da comunicação terapêutica como instrumentos de cuidado de enfermagem ${ }^{(8)}$.

Diante do exposto, o presente estudo pretende apresentar um panorama sobre a inserção da temática do relacionamento terapêutico e da comunicação terapêutica no ensino de graduação em enfermagem, mais particularmente nas universidades públicas do Estado de São Paulo. 


\section{METODOLOGIA}

Neste estudo, realizamos uma pesquisa qualitativa e descritivo-analítica. $\mathrm{O}$ instrumento proposto foi um questionário aplicado aos professores da área de enfermagem psiquiátrica e saúde mental das 9 Escolas de Graduação em Enfermagem públicas do Estado do São Paulo.

Como primeiro procedimento metodológico, encaminhamos o projeto ao comitê de ética da Escola de Enfermagem de Ribeirão Preto-USP, obtendo parecer favorável ao seu desenvolvimento. Posteriormente, encaminhamos um ofício informando o objeto de estudo, objetivos e procedimentos metodológicos da pesquisa para as Escolas de Enfermagem. A intenção era obter a concordância das instituições estudadas, o termo de consentimento livre e esclarecido a ser assinado pelos informantes e o preenchimento do questionário - instrumento desta pesquisa, respeitando os procedimentos éticos previstos na legislação brasileira para pesquisa em seres humanos. O questionário com questões abertas e fechadas acerca do ensino de enfermagem psiquiátrica foi encaminhado às 09 Escolas de Graduação em Enfermagem públicas Estado de São Paulo e 08 Escolas retornaram o questionário preenchido.

Com relação à operacionalização dos dados, realizamos as seguintes etapas: ordenação, classificação e análise final. A ordenação dos dados obtidos através do questionário ocorreu após re-leituras sucessivas de todo o material, agrupamentos e organização dos dados a partir da reflexão sobre o objetivo da pesquisa, resultando em temáticas genéricas discriminadas nos resultados. A classificação dos dados ocorreu a partir de tentativas de determinar o conjunto das informações presentes no questionário, considerando as convergências e divergências centrais verificadas.

Os dados foram agrupados segundo a caracterização do quadro docente e a caracterização do ensino, incluindo disciplinas ofertadas, carga horária, local de realização de aulas práticas, conteúdos abordados e projetos de extensão. Estes dados foram analisados a partir das seguintes categorias operacionais: contextualização da trajetória do portador de transtorno psíquico na história e novos dispositivos de cuidado em saúde mental, tendo-se, como categoria central de análise, o relacionamento terapêutico.

\section{RESULTADOS E DISCUSSÃO}

O relacionamento terapêutico constitui-se em uma tecnologia de cuidado de enfermagem que permite o entendimento das experiências de vida do paciente, o estímulo à sua participação na tomada das decisões terapêuticas e o reconhecimento de paciente e enfermeiro enquanto seres humanos dotados de saberes próprios, limitações pessoais e potencialidades.

Após uma releitura sucessiva e uma reorganização dos dados emergidos dos questionários, fizemos uma classificação da temática do relacionamento terapêutico nas diferentes escolas que participaram do estudo e montamos um quadro, explicitado a seguir:
Relacionamento terapêutico e ensino de enfermagem psiquiátrica e saúde mental: tendências no Estado de São Paulo

Quadro 1- Caracterização dos conteúdos sobre a temática nas instituições e objetivos das atividades práticas. (Ribeirão Preto, 2004)

\begin{tabular}{|c|l|l|l|l|}
\hline INSTITUIÇÃO & DISCIPLINAS & $\begin{array}{l}\text { CONTEÚDOS } \\
\text { ABORDADOS }\end{array}$ & $\begin{array}{l}\text { OBJETIVO DAS } \\
\text { ATIVIDADES } \\
\text { PRÁTICAS }\end{array}$ & $\begin{array}{c}\text { CAMPOS } \\
\text { PRÁTICOS }\end{array}$ \\
\hline 1 & $\begin{array}{l}\text { Enfermagem } \\
\text { Psiquiátrica }\end{array}$ & $\begin{array}{l}\text { Não são } \\
\text { ministrados } \\
\text { conteúdos teóricos } \\
\text { sobre relaciona- } \\
\text { mento terapêutico }\end{array}$ & $\begin{array}{l}\text { Participação nas } \\
\text { atividades } \\
\text { multidisciplinar }\end{array}$ & $\begin{array}{l}\text { Hospital-Dia, } \\
\text { Hospital Geral, } \\
\text { Ambulatório } \\
\text { de Saúde Mental e } \\
\text { CAPS }\end{array}$ \\
\hline 2 & $\begin{array}{l}\text { Enfermagem } \\
\text { Psiquiátrica } \\
\text { Saúde Mental }\end{array}$ & $\begin{array}{l}\text { Relacionamento } \\
\text { interpessoal } \\
\text { enfermeiro-paci- } \\
\text { ente e comunicação }\end{array}$ & $\begin{array}{l}\text { Direcionadas ao } \\
\text { relacionamento } \\
\text { profissional-paciente, } \\
\text { à valorização da } \\
\text { história de vida e à } \\
\text { escuta terapêutica }\end{array}$ & $\begin{array}{l}\text { Psiquiátrico, } \\
\text { Hospital-Geral, } \\
\text { CAPS e CSE }\end{array}$ \\
\hline
\end{tabular}


Luciane Prado Kantorski Leandro Barbosa de Pinho Toyoko Saeki

Maria Conceição B.M. Souza

\begin{tabular}{|c|c|c|c|c|}
\hline INSTITUIÇÃO & DISCIPLINAS & $\begin{array}{l}\text { CONTEÚDOS } \\
\text { ABORDADOS }\end{array}$ & $\begin{array}{c}\text { OBJETIVO DAS } \\
\text { ATIVIDADES } \\
\text { PRÁTICAS }\end{array}$ & $\begin{array}{c}\text { CAMPOS } \\
\text { PRÁTICOS }\end{array}$ \\
\hline 3 & $\begin{array}{l}\text { Enfermagem } \\
\text { Psiquiátrica na } \\
\text { Saúde Mental II } \\
\text { Enfermagem } \\
\text { Psiquiátrica na } \\
\text { Saúde do Adulto II } \\
\text { - Fundamentos do } \\
\text { Processo de } \\
\text { Cuidar } \\
\\
\text { Estágio Curricular } \\
\text { de Enfermagem } \\
\text { Materno In-fantil } \\
\text { e em Saúde } \\
\text { Mental }\end{array}$ & $\begin{array}{l}\text { Relacionamento } \\
\text { interpessoal } \\
\text { enfermeiro-paciente } \\
\text { e comunicação } \\
\text { terapêutica } \\
\text { Tipos de relaciona- } \\
\text { mento interpessoal, } \\
\text { Comunicação Tera- } \\
\text { pêutica e medidas } \\
\text { terapêuticas na } \\
\text { enfermagem (apoio } \\
\text { e limites) } \\
\text { Relacionamento In- } \\
\text { terpessoal e medi- } \\
\text { das terapêuticas na } \\
\text { enfermagem }\end{array}$ & $\begin{array}{l} \\
\text { Baseiam-se na obser- } \\
\text { vação e no relaciona- } \\
\text { mento interpessoal, } \\
\text { além de outras ativi- } \\
\text { dades como anota- } \\
\text { ções de enfermagem } \\
\text { e planejamento de } \\
\text { assistência }\end{array}$ & $\begin{array}{l}\text { Hospital } \\
\text { Psiquiátrico, } \\
\text { Hospital-Geral, } \\
\text { CAPS e } \\
\text { Ambulatório de } \\
\text { Saúde Mental }\end{array}$ \\
\hline 4 & $\begin{array}{l}\text { Enfermagem } \\
\text { Psiquiátrica }\end{array}$ & $\begin{array}{l}\text { Relacionamento } \\
\text { Interpessoal } \\
\text { Enfermeiro } \\
\text {-Paciente }\end{array}$ & $\begin{array}{l}\text { Hospital psiquiátrico, } \\
\text { com ênfase no rela- } \\
\text { cionamento } \\
\text { interpessoal }\end{array}$ & $\begin{array}{l}\text { Hospital } \\
\text { Psiquiátrico }\end{array}$ \\
\hline 5 & $\begin{array}{l}\text { O currículo é } \\
\text { estruturado em } \\
\text { unidades }\end{array}$ & $\begin{array}{l}\text { Produção social da } \\
\text { saúde com o tema } \\
\text { entrevista e } \\
\text { comunicação } \\
\text { (Unidade 4) }\end{array}$ & $\begin{array}{l}\text { Embasada no relaci- } \\
\text { onamento interpes- } \\
\text { soal com o paciente. } \\
\text { Direcionada confor- } \\
\text { me as necessidades } \\
\text { dos alunos ou dos } \\
\text { campos de estágio }\end{array}$ & $\begin{array}{l}\text { Hospital Geral, } \\
\text { Ambulatório de } \\
\text { Saúde Mental e } \\
\text { Unidade Básica de } \\
\text { Saúde }\end{array}$ \\
\hline 6 & $\begin{array}{l}\text { Enfermagem } \\
\text { Psiquiátrica }\end{array}$ & $\begin{array}{l}\text { Relacionamento } \\
\text { Terapêutico } \\
\text { (Unidade II) }\end{array}$ & $\begin{array}{l}\text { Enfocam a relação } \\
\text { terapêutica e a assis- } \\
\text { tência de enferma- } \\
\text { gem em geral }\end{array}$ & $\begin{array}{l}\text { Hospital } \\
\text { Psiquiátrico e } \\
\text { visitas a serviços } \\
\text { substitutivos }\end{array}$ \\
\hline 7 & $\begin{array}{l}\text { Enfermagem em } \\
\text { Saúde Mental I } \\
\text { Enfermagem na } \\
\text { Saúde do Adulto e } \\
\text { do Idoso } \\
\text { Assistência de } \\
\text { Enfermagem a } \\
\text { Clientes de Alto } \\
\text { Risco }\end{array}$ & $\begin{array}{l}\text { Cuidados de enfer- } \\
\text { magem com ênfase } \\
\text { no relacionamento } \\
\text { interpessoal e na } \\
\text { comunicação } \\
\text { terapêutica }\end{array}$ & $\begin{array}{l}\text { Prioriza a } \\
\text { subjetividade do } \\
\text { sofrimento mental e } \\
\text { a psicopatologia }\end{array}$ & $\begin{array}{l}\text { Centro de } \\
\text { Reabilitação em } \\
\text { Dependência } \\
\text { Química, Unidade } \\
\text { Básica, CAPS, } \\
\text { Hospital Geral e } \\
\text { Hospital-Dia }\end{array}$ \\
\hline 8 & $\begin{array}{l}\text { Enfermagem em } \\
\text { Saúde Mental } \\
\text { Enfermagem } \\
\text { Psiquiátrica }\end{array}$ & $\begin{array}{l}\text { Relacionamento } \\
\text { interpessoal enfer- } \\
\text { meiro-paciente, } \\
\text { papel terapêutico } \\
\text { do enfermeiro e } \\
\text { ambiente } \\
\text { terapêutico }\end{array}$ & Não informado & $\begin{array}{l}\text { Hospital } \\
\text { Psiquiátrico e } \\
\text { Geral, incluindo } \\
\text { enfermarias de } \\
\text { risco de crise } \\
\text { (oncologia, } \\
\text { ginecologia e } \\
\text { cirurgia vascular) }\end{array}$ \\
\hline
\end{tabular}


Pudemos observar que a instituição 1 figura entre as exceções das escolas que não ministram conteúdos sobre relacionamento terapêutico. Nos dados informados, apenas são ministrados conteúdos que priorizam o atendimento de enfermagem nas diversas psicopatologias e enfermidades mentais. As atividades práticas nos campos de estágio são direcionadas à projeção de um plano de assistência com participação multidisciplinar.

Contudo, notamos que a grande maioria das escolas de enfermagem em São Paulo preconiza, na formação profissional do enfermeiro, conteúdos sobre relacionamento interpessoal e comunicação interpessoal. As instituições 2, 3, 4, 5, 6, 7 e 8 , por exemplo, fazem destaque aos conteúdos de relacionamento terapêutico nos currículos dos cursos de enfermagem. Consideram-no também como um instrumento teórico-prático de trabalho e ensino ao enfocarem e priorizarem o atendimento aos pacientes embasado na relação interpessoal.

A instituição 2 aborda o conteúdo de relacionamento terapêutico e comunicação terapêutica nas disciplinas de Enfermagem Psiquiátrica e Introdução à Saúde Mental. O atendimento é direcionado ao relacionamento profissionalpaciente, à valorização da história de vida do portador de sofrimento psíquico e à escuta terapêutica.

Da mesma forma, a instituição 3 trabalha com a temática em diferentes disciplinas da graduação, não somente nas disciplinas de Enfermagem Psiquiátrica. Ressaltamos aqui a preocupação com o relacionamento interpessoal, que faz parte das disciplinas de Enfermagem Psiquiátrica na Saúde do Adulto II - Fundamentos do Processo de Cuidar e Estágio Curricular de Enfermagem Materno-Infantil e em Saúde Mental. O foco de trabalho nos campos de estágio se dá na observação e no relacionamento interpessoal, além de outras atividades, como as anotações de enfermagem e o planejamento da assistência.

As instituições 4, 5 e 6 também expressam suas preocupações com a formação do enfermeiro em nível de relacionamento interpessoal. As escolas mencionam o relacionamento interpessoal enfermeiro-paciente e a comunicação como conteúdos integrantes da formação teórico-prática do profissional.

A instituição 7 faz referências à temática nas disciplinas de Enfermagem em Saúde Mental I, Enfermagem na Saúde do Adulto e do Idoso e Assistência de Enfermagem a Clientes de Alto Risco. Com relação à disciplina de Enfermagem em Saúde
Mental I, os cuidados prestados pelos alunos têm por base os princípios do relacionamento terapêutico e da comunicação terapêutica, embora a prioridade da disciplina seja as psicopatologias, a assistência de enfermagem nos transtornos mentais e as políticas de saúde mental.

A instituição 8 evidencia conteúdos teóricos sobre relacionamento terapêutico enfermeiro-paciente, enfatizando também o papel terapêutico do enfermeiro e o ambiente terapêutico como promotores de saúde mental.

Fazendo um resgate histórico-social das práticas no campo de enfermagem psiquiátrica, destaca-se que a partir da década de 70, o ensino de enfermagem psiquiátrica nas escolas de enfermagem brasileiras se modifica para abarcar as mudanças que emergiam diante das transformações que a reforma psiquiátrica conferia ao modelo de atenção psiquiátrico. O relacionamento terapêutico surge como alternativa teórico-metodológica para a prestação de assistência de enfermagem humanizada e centrada nas necessidades da pessoa que vivencia uma situação de sofrimento psíquico $^{(9)}$.

Os estudos de Hildegard Peplau ${ }^{(10)}$ e Joyce Travelbee ${ }^{(11)}$ foram importantes referenciais para subsidiar a assistência de enfermagem e para estudos posteriores sobre a temática ${ }^{(12-15)}$. Segundo as autoras, a retomada das relações com o mundo externo do portador de transtorno psíquico está condicionada à existência de um elo de ligação entre o enfermeiro e o paciente. Esse encontro se torna potencial meio para a troca de experiências, para o saneamento das dúvidas, para a emergência de recursos internos de enfrentamento dos problemas e, principalmente, para fazer com que o indivíduo se reconheça e participe integralmente de seu tratamento com relativa independência.

Os estudos de Hildegard Peplau na década de 50 somente foram desenvolvidos segundo os princípios da psicoterapia e da psiquiatria psicoterápica, consolidando o relacionamento terapêutico como o primeiro instrumento de sistematização em enfermagem psiquiátrica que fora realizado por uma enfermeira $^{(13)}$.

O relacionamento terapêutico enfermeiro-paciente se torna um substancial instrumento de ajuda e entendimento do outro. Cada paciente possui comportamentos específicos e diferentes maneiras de pensar e agir. A enfermagem deve se adaptar à singularidade do ser humano, compreendendo-o em toda sua trajetória de vida e planejando a assistência de acordo com suas necessidades ${ }^{(15-16)}$.
Relacionamento terapêutico e ensino de enfermagem psiquiátrica e saúde mental: tendências no Estado de São Paulo 
Luciane Prado Kantorski Leandro Barbosa de Pinho Toyoko Saeki

Maria Conceição B.M. Souza
O terapeuta, para exercer com afinco o relacionamento terapêutico, deve ser autêntico, sincero, empático e demonstrar comprometimento com a realidade do cliente ${ }^{(17)}$. A autenticidade, a sinceridade e o comprometimento permitem ao terapeuta desvelar um profundo entendimento da vida íntima do cliente para que possa ajudá-lo:

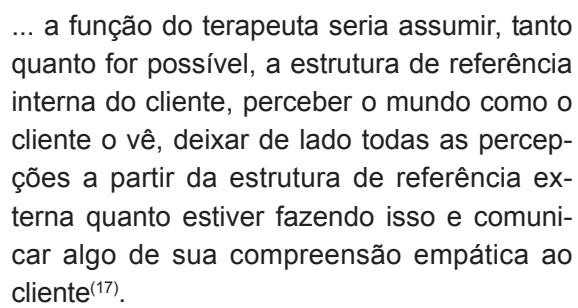

Empatia é imprescindível no processo de relacionamento terapêutico. Ser empático significa experimentar o cliente, ou seja, imergir nele e compartilhar de suas vivências e atitudes, respeitando-o em suas individualidades. O terapeuta deve perceber todos os ódios, os medos, as dúvidas e as esperanças através de uma identificação empática, não simplesmente emocional, sem, contudo, experimentar ele próprio esses sentimentos como se também fossem parte dele ${ }^{(17)}$.

Entendemos que o relacionamento terapêutico vem-se constituindo ao longo da história como um saber-fazer da enfermagem psiquiátrica que busca a contínua humanização do atendimento ao portador de sofrimento psíquico. É um contrato de envolvimento entre o terapeuta e o paciente/família que particulariza nuanças, permite reconhecer a singularidade de cada ser humano, suas relações sociais, perspectivas, fragilidades e viabilidades, bem como possibilita o resgate da autoconfiança, da autovalorização e da re-inserção no mundo externo.

Entretanto, o relacionamento terapêutico é um instrumento de cuidado intersubjetivo. Isso significa que ele trabalha não somente com as necessidades psicossociais do paciente, como também com as necessidades do educandocuidador. A ação recíproca que existe no contato tera-pêutico se movimenta numa perspectiva dialética, em que ocorrem transformações intra e interpessoais. Se

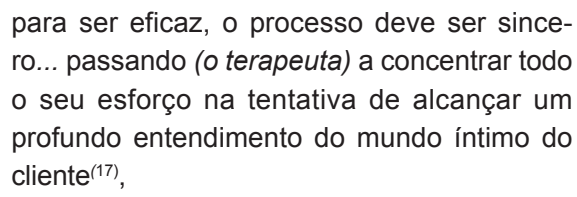

o cuidador profissional (no caso do ensino o aluno) também carece de preparo técnico-científico para exercê-lo com efetividade. Tal justificativa se deve ao fato de justamente permitir a ele se conhecer e a trabalhar primeiro com as suas incompatibilidades, limitações e potencialidades para que possa se voltar à interpretação e ajuda do paciente também dotado das mesmas condições.

Quando o aluno entra no campo da psiquiatria e saúde mental, ele não somente se depara com a complexidade do padecer psíquico, como também se defronta com suas próprias potencialidades e limitações perante a situação. O educando passa a notar que o cuidado psiquiátrico não pode ser organizado em uma seqüência de tarefas, carecendo estabelecer-se o vínculo com o paciente para, a partir daí, direcionar um plano de assistência. Desse modo, o aluno precisa de tempo, acompanhamento e sistemática orientação teórico-prática. A escuta, o manejo, o vínculo e o cuidado são conceitos complexos em se tratando de saúde mental porque exigem mais que assimilação. Vão requerer que o indivíduo se disponha a trabalhar limitações e dificuldades pessoais, relacionamento, entre outras ${ }^{(18)}$.

Essa preocupação com o próprio aluno é evidenciada pela instituição 5, que particulariza o ensino do relacionamento terapêutico de forma a estruturar o bloco teórico conforme as necessidades evidenciadas pelos alunos ou oportunidades oferecidas pelos campos de estágios. Acreditamos que isso se configura como uma flexibilidade visível da escola em proporcionar, ao educando, primeiro o conhecimento e reconhecimento de si para que, a posteriori, possa trabalhar visando ao conhecimento do outro.

Precisamos ter em mente que alunos são pessoas dotadas de dificuldades e limitações tanto quanto os pacientes. Devemos proporcionar espaços para a reflexão pessoal e a discussão contínua e sistematizada dessas dificuldades, com vistas à formação de um enfermeiro mais acolhedor e continente com o sofrimento do outro. $\mathrm{O}$ aprendizado nas escolas realmente deve ser pautado evidenciando-se essas particularidades. Deve também mostrar que as dificuldades, tanto pessoais como interpessoais, podem atrapalhar ou até mesmo impedir a ajuda terapêutica. Mesmo assim, deve salientar que no processo de interação com o outro, em algum momento do relacionamento, ela também se tornou terapêutica.

Compreendemos que as escolas de enfermagem, ao abordarem o relacionamento terapêutico em suas disciplinas, transformam-se em poderosos instrumentos de disseminação do paradigma humanizador e pautado no respeito ao ser huma- 
no em sua totalidade, em qualquer condição biopsicossocial. O resgate das concepções teórico-tecnológicas sobre relacionamento terapêutico oferece o fomento à formação de profissionais humanos, sensíveis e reconhecedores das limitações de seus pacientes, como também das suas próprias limitações enquanto terapeutas.

Reportando-nos às instituições 3 e 7 , um fato interessante que encontramos na análise foi a abordagem do relacionamento terapêutico em outras disciplinas, além da enfermagem psiquiátrica e saúde mental. Nessas instituições, por exemplo, os conteúdos são ministrados em diferentes áreas de concentração, como na saúde da mulher, na saúde do idoso e nas disciplinas que lidam com as enfermidades críticas.

Com relação à instituição 3, identificamos que ela menciona o relacionamento terapêutico como temática abordada nas disciplinas de Enfermagem Psiquiátrica na Saúde do Adulto II - Fundamentos do Processo de Cuidar e em Estágio Curricular de Enfermagem Materno Infantil e em Saúde Mental.

A instituição 7, por sua vez, aborda o relacionamento terapêutico nas disciplinas de Enfermagem na Saúde do Adulto e do Idoso e Assistência de Enfermagem a Clientes de Alto Risco. Conforme exposto, o atendimento é voltado à compreensão do paciente e no estabelecimento do vínculo que é primazia para o relacionamento terapêutico.

Em um estudo realizado com familiares de pacientes internados na unidade de emergência, constatou-se que a família sofre diante do desligamento temporário com o seu familiar, evidenciando-se condições para o surgimento do sofrimento psíquico. O limitado envolvimento emocional por parte da enfermagem potencializa esse sofrimento dos familiares, bem como estigmatiza a unidade de emergência como palco de inquietações e insegurança emocional para familiares e pacientes. Assim, entende-se que o cuidado humanizado na unidade ainda é incipiente, havendo a necessidade de a enfermagem mediar as relações frente ao desligamento temporário imposto a pacientes e familiares, apoderando-se do relacionamento terapêutico e da comunicação terapêutica como instrumentos de cuidado $^{(19)}$.

Em tempos de tecnologização do cuidado, é particularmente importante o resgate de uma concepção antropológico-holística do ser humano, entendendo-o em toda a sua complexidade e cuidando do sofrimento gerado pelas mais diversas situações em todas as suas dimensões, seja ela física, psíquica, social, emocional e espiritual. Cabe aos profissionais de saúde desenvolver competências que lhes aproximem das práticas humanizadas, em que o sofrer possa ser remanejado e redimensionado ${ }^{(1)}$.

A abordagem da temática do relacionamento terapêutico pelas instituições 3 e 7 em outras disciplinas de formação do enfermeiro reitera que o sofrimento psíquico pode tornar-se manifestação expressiva em qualquer fase do ciclo vital. O acometimento de uma doença, por exemplo, em qualquer etapa da vida, pode ser uma dessas manifestações. $\mathrm{O}$ estudo que ora mencionamos corrobora nossas argumentações. Assim, remarcamos que o relacionamento terapêutico é excelente ferramenta promotora de saúde mental em qualquer situação que exija acompanhamento intensivo ou semi-intensivo da enfermagem, seja na área da psiquiatria ou saúde mental, ou em qualquer outra área do conhecimento da profissão.

\section{CONSIDERAÇÕES FINAIS}

Pudemos constatar que as Escolas de Graduação em Enfermagem de caráter público do Estado de São Paulo, em sua grande maioria, abordam conteúdos sobre relacionamento terapêutico e comunicação terapêutica em seus currículos. Verificamos que algumas escolas ainda priorizam o ensino de relacionamento terapêutico e comunicação terapêutica não somente nas disciplinas de saúde mental e enfermagem psiquiátrica, mas também em outras disciplinas de formação do enfermeiro. A flexibilidade apontada permite-nos inferir que as escolas têm necessidade de ressaltar o relacionamento terapêutico como uma contribuição da saúde mental que pode ser utilizada como tecnologia de cuidado em outras áreas do conhecimento da profissão, como na saúde da mulher, na saúde do idoso e nas enfermidades que exigem cuidados críticos.

Entendemos que a preocupação das Escolas em ministrar conteúdos sobre relacionamento terapêutico e comunicação terapêutica permite o resgate da enfermagem enquanto profissão que lida principalmente com o sofrimento humano e não somente com sua doença. Cria-se, portanto, um cenário de reflexão, discussão, produção e reprodução de saberes e práticas pautados no paradigma humanizador, ressaltando-se o respeito à dignidade do ser humano que padece em toda sua complexidade biopsicossocial, constituído de limitações, potencialidades, desejos, necessidades e relações interpessoais.
Relacionamento terapêutico e ensino de enfermagem psiquiátrica e saúde mental: tendências no Estado de São Paulo 
Luciane Prado Kantorski Leandro Barbosa de Pinho Toyoko Saeki

Maria Conceição B.M. Souza

\section{REFERÊNCIAS}

(1) Pessini L. Humanização da dor e sofrimento humanos no contexto hospitalar. Bioética [periódico online] 2002; 10:2. Disponível em:http://www. emportalmedico.org/revista/bioética/bio10v2/ simpósio1.htm (16 mar. 2003)

(2) Silva AL, Fonseca RMGS. Os nexos entre concepção do processo saúde/doença mental e as tecnologias de cuidados. Rev Lat-Am Enferm 2003; 11(6):800-6.

(3) Caplan G. Princípios de psiquiatria preventiva. Rio de Janeiro: Zahar; 1980.

(4) Kohlrausch E. O modelo assistencial clínico e algumas possibilidades de fazer diferente. Rev Gaúcha Enferm 1999; 20 (n. esp):70-85.

(5) Arantes EC. O ensino de relacionamento terapêutico em enfermagem psiquiátrica. Rev Bras Enferm 1973; 26(6):439-46.

(6) Fraga MNO, Damasceno RN, Calixto MLG. Ensino de relacionamento terapêutico em enfermagem psiquiátrica: dificuldades e perspectivas. Rev Bras Enferm 1986; 39(2/3):94-102.

(7) Stefanelli MC. Ensino de técnica de comunicação terapêutica enfermeira-paciente parte I. Rev Esc Enferm USP 1986; 20(2):161-83.

(8) Kantorski LP, Pinho LB, Schrank G. O relacionamento terapêutico e o cuidado em enfermagem psiquiátrica e saúde mental. Rev Enferm UERJ 2003; 12(2):201-7.

(9) Souza MCM, Alencastre MB. Produção de enfermagem psiquiátrica no Brasil: 1932-1993. Rev Bras Enferm 1999; 52(2):271-82.
(10) Peplau HE. Relaciones interpersonales en enfermería. Barcelona: Salvat; 1990.

(11) Travelbee J. Intervención en enfermería psiquiátrica. Colombia: Carvajal; 1979.

(12) Furegato ARF. Relações interpessoais terapêuticas na enfermagem. Ribeirão Preto: Escala; 1999.

(13) Campos CMS, Barros S. Reflexões sobre o processo de cuidar da enfermagem em saúde mental. Rev Esc Enferm USP 2000; 34(3):271-6.

(14) Stefanelli MC. Comunicação em enfermagem: teoria, ensino e pesquisa. [tese] São Paulo (SP): Escola de Enfermagem da USP; 1990.

(15) Stefanelli MC. Relacionamento terapêutico enfermeira-paciente. Rev Esc Enferm USP 1983; 17(1):39-45

(16) Nunes MGB, Rocha RM, Santos MLSC, Silva NF. Desenvolvimento de enfermagem planejada em psiquiatria. Rev Bras Enferm 1986; 39 (2/3):46-50.

(17) Rogers CR. Terapia centrada no cliente. São Paulo: Martins Fontes; 1992.

(18) Kantorski LP, Silva GB. Ensino de enfermagem e reforma psiquiátrica. Pelotas: Editora Universitária UFPel; 2001.

(19) Pinho LB, Kantorski LP. Refletindo sobre o contexto psicossocial de famílias de pacientes internados na unidade de emergência. Cienc y Enferm 2004; 10(1):67-77. 